\title{
IMPLEMENTING TOTAL PRODUCTIVE MAINTENANCE (TPM) IN MALAYSIAN MANUFACTURING ORGANISATION: AN OPERATIONAL STRATEGY STUDY
}

\author{
One Yoon Seng \\ Muhamad Jantan \\ T. Ramayah \\ School of Management, Universiti Sains Malaysia
}

\begin{abstract}
-
Abstract

Total Productive Maintenance (TPM) has been recognized as one of the significant operation strategy to regain the production losses due to equipment inefficiency. Many organizations have implemented TPM to improve their equipment efficiency and to obtain the competitive advantage in the global market in terms of cost and quality. In the implementation of TPM in a manufacturing organization, both Human-oriented and Process-oriented Strategy has been identified as critical success factors. This paper, therefore, focuses on the two TPM operational strategies, which is posited, will improve the extent of TPM implementation in manufacturing organizations. This study is an attempt to look at the TPM implementation from the perspective of a developing country such as Malaysia.
\end{abstract}

Keywords: total productive maintenance, strategy, manufacturing, Malaysia

\section{INTRODUCTION}

Efficiency and effectiveness of equipment plays a dominant role in modern manufacturing industry to determine the performance of the organizational production function as well as the level of success achieved in the organization. For more then two decades, the development of the Malaysian manufacturing sector had registered an excellent performance and attracted a large number of foreign capital investments to this country. These excellent performances have enabled the Malaysian manufacturers to enjoy an important competitive advantage in the global market, especially in terms of cost and quality. However, as time passed, the impact of equipment efficiency has become more and more critical as the widespread utilization and application of highly sophisticated and automated machines in the industry increases. The maintenance of these complicated equipment and machines thus became very crucial and costly to manufacturers. Many organizations began to realize that the continuity of this excellent performance must be supported by a strong backbone of efficient and effective equipment. Traditional maintenance technicians are regarded as passive and non-productive to the current production function. Hence, implementing Total Productive Maintenance (TPM) in the manufacturing industry has emerged as an important operational strategy to overcome the production losses due to equipment inefficiency. TPM is an innovative approach, which holds the potential for enhancing the efficiency and effectiveness of production equipment by taking advantages of abilities and skills of all individuals in the organization. TPM focuses on maximizing the Overall Equipment Efficiency (OEE) with involvement of each and everyone in the organization. It will not only establish a complete maintenance system, but also aims to improve the maintenance skills and knowledge among the shop floor operators. Now, TPM and its implications received prestigious worldwide recognition in achieving the ultimate Zero Defects and Zero Breakdown targets. 
This paper aims to assess the optimum TPM operational strategy which will increase the chances of a successful TPM implementation within a manufacturing organization in a developing country perspective. At the same time, few implementation issues such as motivators, critical success factors and factors that inhibit the extent and success of TPM implementation in the Malaysian manufacturing sector will also be discussed.

\section{LITERATURE REVIEW}

\subsection{Human-oriented Strategy}

Human-oriented strategy is, generally, strategies that actively involve human administrative application of management methods in achieving high extent of TPM. Three important aspects that are often discussed as the core of Human-oriented strategy are: (1) Top management commitment and leadership, (2) Total Employee Involvement, and (3) Training and Education.

\subsubsection{Top Management Commitment and Leadership}

The role of top management's commitment and leadership has been frequently emphasized in many literatures to have the decisive influence over successful TPM implementation (Tsang \& Chan, 2000). TPM requires a drastic change in the traditional mindset of work culture and maintenance approaches. However at the present moment, high resistance is often encountered from the shop floor operators and as well as the maintenance personnel. To this extent, active top management support is crucial to overcome such resistance, especially during the transition period (Fredendall, 1997). Bamber et al. (1999) wrote that the major obstacle in implementing TPM in UK was the lack of top management commitment to follow through which resulted in many organizations to struggle when attempting to implement TPM. Patterson (1996) explained that to successfully implement TPM, an organization must be led by top management that is supportive understanding and committed to the various kinds of TPM activities. Top management has the primary responsibility of preparing a suitable and supportive environment before the official kick-off of TPM within their organization. This may include resources allocation and training and education provided to the middle management level as well as the production floor operators. Nakajima (1989) stated that the top management's primary responsibility is to establish a favorable environment where the work environment can support autonomous activities.

\subsubsection{Total Employee Involvement}

While top management commitment and leadership is essential for TPM success, it is not sufficient on its own. TPM embraces empowerment to production operators establishing a sense of ownership in their daily operating equipment (Tsang \& Chan, 2000). This sense of ownership is an important factor that underpins TPM to its continual success with every operator being responsible to ensure her own machine is clean and maintained. It involves the employees to have a common understanding of the basic principles of TPM. The importance of total employee involvement is based on the beliefs that shop floor operators have the most hands-on experience with the machines they operate daily. Thus, TPM demands active participation from the shop floor operators in the continuous improvements activities, cross-functional teamwork, work suggestion schemes (Nakajima, 1989). High level of maintenance awareness and simple routine maintenance tasks are integrated into their daily duties and the final mission ahead is to achieve profitable Autonomous Maintenance by operators. TPM accomplished the maximization of equipment effectiveness through total employee participation and incorporated the use of Autonomous Maintenance in the small group activities to improve on the equipment reliability, maintainability and productivity (Chen, 1997). 


\subsubsection{Training and Education}

Blanchard (1997) pointed out that training and educational issues had become one of the critical factors to establish successful TPM implementation, where proper education begin as early as during the TPM introduction and initial preparation stages. The entire workforce in the organization need to acquire new knowledge, skill and abilities related to TPM. Thiagarajan and Zairi (1997) further addressed that education and training is the single most important factor once the necessary commitment has been assured and had become a long-term strategy in the planning schedule to obtain aspirations and skills. Further implementation of TPM sees the training to be essential to the implementation and work performance.

\subsection{Process-oriented Strategy}

While Human-oriented Strategy is important to prepare the foundation prior to implementing TPM, Process-oriented Strategy plays an important role in the next part to achieve a successful TPM implementation in the organization. Process-oriented Strategy includes all kinds of technical approaches to maximize the overall equipment efficiency through quantitatively, increasing the equipment availability and qualitatively, eliminating all production losses that resulted from inefficient equipment (Nakajima, 1989). The primary goal of TPM is to achieve the ultimate target of Zero Loss and Zero Breakdown so that all equipments are performing at its optimal condition. The production function performance is diminished by inefficient equipment that generates losses in terms of failureloss, performance-loss or defect-loss. In detail, such losses can be refined as Equipment breakdown; Setup and adjustment time loss; Idling and minor stoppages; reduced performance rate (slower speed); Process failure (defects) and reworks and Startup time losses. Therefore, one of the major features of Process-oriented Strategy emphasizes on hands-on and practical approaches to identify and quantify all the above losses in the production floor (Suzuki, 1994). The sequential step-wise procedure of Process-oriented Strategy begins with:

i. $\quad$ identifying failures or losses and analyze causes;

ii. setting improvements to eliminate failures and losses;

iii. Confirming and consolidating results.

The immediate subsequent action after identifying and quantifying the equipment losses is to stratify and analyze the relevant root causes. Some of the analytical methods that had been developed and widely deployed to promote the thorough and systematic elimination of defects in Process-oriented Strategy are PM analysis, Fault-tree analysis (FTA), Failure Mode and Effect analysis (FMEA) and so on (Suzuki, 1994). With all failure phenomena being clearly described, improvements become crucial in the second stage to eliminate these pertinent causes. Improvement plans had to be, in balance, carried out on both equipment and process. Shimbun (1995) stated that, for improvement of equipment, the very basic activities are to restore all forms of deterioration in the equipment and establishing the basic condition.

Thereupon, Process-oriented Strategy should concentrate on process improvement to its optimal condition. Any human factors or operations which lead to the accelerated deterioration of the equipment are eliminated as well as improved in terms of operation and maintenance skills. In addition to the process improvement, the payoff from the simplification of operation has many direct advantages as well as various useful spin-off benefits in terms of increased equipment efficiency (Suzuki, 1994). The cycle of Process-oriented Strategy is completed by counter-checking and consolidating the positive results of improvements that was being implemented. To these circumstances, equipment standardization and work instruction controls are extensively carried out across the board of production floor. 


\section{PROBLEM STATEMENT}

Introducing TPM in a developing country, such as Malaysia, is still considered a major challenge due to several non-conducive environments in the adoption and implementation process. Lack of commitment and leadership from top management has always been discussed as one of the main factors that inhibit the implementation of TPM. On the other hand, resistance from the employee involved in the TPM program is also regarded as another major reason that explains why TPM fails in many local organizations. Employees refused to endure extra maintenance responsibilities without any rewards, recognition or compensation. Lack of proper and adequate training and education about TPM also contributed another significant percentage to the pitfalls of TPM implementation in a developing country.

\section{RESEARCH METHODOLOGY}

A structured survey approach has been used as the research strategy in this study where questionnaires regarding the implementation of TPM were distributed to industrial manufacturers. The questionnaires was divided into several sections to capture all the relevant data and information such as organization profile, TPM background, extent of current TPM activities, success of TPM and technological complexity in the organization. The target respondents of this study are the industrial manufacturers (operation/maintenance managers) in Malaysia, especially from the north (Penang, Prai and Kulim industrial areas) and from the central region (industrial estates of Shah Alam, Puchong and Petaling Jaya). Sample organizations selected from the FMM Directory of Malaysian Industry and involved in various manufacturing products from consumer electrical \& electronics products, food \& beverage, medical \& health care products, precision tooling \& machining, rubber and plastic based products, semiconductors and so on.

\section{THEORETICAL FRAMEWORK}

Based upon the literature review on TPM implementation and its practices, a research framework of Implementing TPM in a Manufacturing Organization is developed. The main purpose of this study is focused on the TPM Operational Strategy and its inter-relationship with other Extent of TPM Implementation. The research framework is illustrated in Figure 1 below.

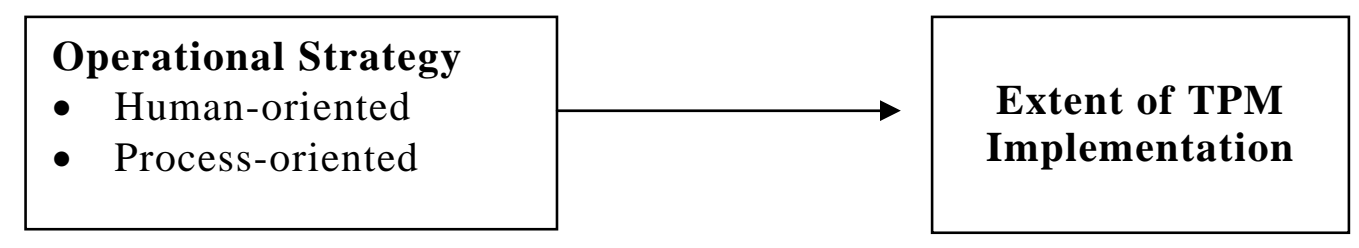

Figure 1 Research Framework

\subsection{Hypothesis Development}

Three hypotheses have been generated in this study to test the relationship of the research framework that has been elaborated earlier. It will test the relationship, especially, between the Operational Strategy and Extent of TPM Implementation.

In the literature review it has been postulated that organizations, which extensively carried out Human-oriented strategy while implementing TPM, would have a higher Extent of TPM within the organization. Basically, when more efforts are devoted to Human-oriented strategy, such as prominent commitment and leadership from the top management, total involvement from all employees will, definitely, result in a higher achievement of TPM. The more an organization implements the Process-oriented strategy (example, process \& equipment improvement, equipment standardization, re-layout, etc.); the maintenance knowledge becomes higher and the Extent of TPM 
implementation will be enhanced. However, the Human-oriented strategy has a greater impact compared to the Process-oriented strategy towards the Extent of TPM implementation. In brief, the three hypotheses of this study are summarized as follows:

$H_{1}$ : $\quad$ Extent of Human-oriented strategy will be positively related to Extent of TPM implementation.

$\mathrm{H}_{2}$ : $\quad$ Extent of Process-oriented strategy will be positively related to Extent of TPM implementation.

$\mathrm{H}_{3}$ : $\quad$ Human-oriented strategy has greater impact on Extent of TPM level then Process-oriented strategy.

\section{FINDINGS}

The following table provides the results of multiple regression analysis that was carried out on both Human-oriented Strategy and Process-oriented Strategy towards the Extent of TPM as dependent variable to test $\mathrm{H}_{1}$ and $\mathrm{H}_{2}$.

Table 1

Regression result for Operational Strategy and Extent of TPM Implementation

\begin{tabular}{lc}
\hline Variables & Beta \\
\hline General Training & $0.205^{\star \star}$ \\
Top Executive Commitment & 0.040 \\
Maintenance Training & $0.271^{\star \star *}$ \\
Supportive TPM environment & 0.071 \\
Workplace Improvement & $0.197^{\star}$ \\
\hline $\mathrm{R}^{2}$ & 0.32 \\
Adj. R & 0.28 \\
$\mathrm{~F}$ Value & $8.47^{\star *}$ \\
\hline${ }^{*} \mathrm{p}<0.05,{ }^{*} \mathrm{p}<0.01$ &
\end{tabular}

The $\mathrm{F}$ value of 8.473 , which is significant at .000 , denotes that there is a significant relationship between Operation Strategy and Extent of TPM implementation. The $\mathrm{R}^{2}$ value of 0.32 indicates that Operation Strategy can together explain $32 \%$ of the variation in the Extent of TPM implementation. A closer look at the coefficients indicate that the above significant relationship is contributed by three variables, the General Training $(\beta=0.205, \quad p<0.05)$, Maintenance Training $(\beta=0.271, p<0.01)$ and Workplace Improvement $(\beta=0.197, p<0.1)$. The positive sign of beta values shows that there is a significant positive relationship between the General Training, Maintenance Training and Workplace Improvement and Extent of TPM Implementation. Both General Training and Maintenance Training are variables that represent the Human-oriented Strategy, thus $\mathrm{H}_{1}$ is partially supported. For Workplace Improvement which is related to Process-oriented Strategy, the significant beta value gives support for $\mathrm{H}_{2}$.

Table 2 and 3 below displays the results of the 2 stage multiple regression of Humanoriented Strategy and Process-oriented Strategy to determine their respective impact towards the extent of TPM implementation, which was stated in $\mathrm{H}_{3}$. 
Table 2

Two stage regression analyses with Human-oriented strategy entered first in the model

\begin{tabular}{lccccc}
\hline & $\mathbf{R}$ & $\mathbf{R}^{\mathbf{2}}$ & Adjusted $\mathbf{R}^{\mathbf{2}}$ & $\Delta \mathbf{R}^{\mathbf{2}}$ & $\mathbf{F}$ \\
\hline Model 1: & 0.54 & 0.29 & 0.26 & 0.29 & $9.40^{\star \star}$ \\
Model 2: & 0.57 & 0.32 & 0.28 & 0.03 & 3.66 \\
\hline
\end{tabular}

Predictors Model 1: $\quad$ Supportive TPM environment, General Training, Maintenance Training, Top Executive Commitment

Predictors Model 2: $\quad$ Supportive TPM environment, General Training, Maintenance Training, Top Executive Commitment, Workplace Improvement

Dependent variable: Extent of TPM.

Table 3

Two stage regression analyses with Process-oriented strategy entered first in the model

\begin{tabular}{|c|c|c|c|c|c|}
\hline & $\mathbf{R}$ & $\mathbf{R}^{2}$ & Adjusted $R^{2}$ & $\Delta \mathbf{R}^{2}$ & $F$ \\
\hline Model 1: & 0.41 & 0.17 & 0.16 & 0.17 & $18.64^{* *}$ \\
\hline Model 2: & 0.57 & 0.32 & 0.28 & 0.15 & $5.12^{* *}$ \\
\hline $\begin{array}{l}\text { Predictors Model 1: } \\
\text { Predictors Model 2: }\end{array}$ & $\begin{array}{l}\text { Wor } \\
\text { Wor } \\
\text { Mai }\end{array}$ & $\begin{array}{l}\text { Impro } \\
\text { Impro } \\
\text { e Tra }\end{array}$ & - Top Executiv & nitmer & \\
\hline
\end{tabular}

The additional impact of Process-oriented Strategy (represented by Workplace Improvement) into Model 2 is not significant at 5\% level and only accounted for 3\% of the variance of the extent of TPM implementation (Table 1). On the other hand, the additional impact from Human-oriented Strategy into Model 2 is found to add $16 \%$ to the total variance of the extent of TPM implementation. Therefore, Human-oriented Strategy has a greater impact on the Extent of TPM implementation then the Process-oriented Strategy. Thus $\mathrm{H}_{3}$ is supported.

\section{DISCUSSION}

\subsection{Impact of Human-oriented Strategy}

The findings show that there is a positive relationship between human-oriented strategy and the extent of TPM implementation. This can be related to the emphasis that the extent of TPM implementation mainly requires new system development and adoption of a new strategy to the organisation itself and its success depends exclusively on work culture, organisational practices and so on, which are human related issues. The findings that training is a significant determinant of TPM implementation supports the works of Blanchard (1997) who pointed out that training and educational issues become the critical factors to establish successful TPM implementation. It is also supported by the study of Thiagarajan and Zairi (1997) singled out education and training as the important drivers after the initial commitment to carry out the TPM implementation has been mad.

\subsection{Impact of Process-oriented Strategy}

The importance of Process-oriented Strategy is also a basic part of the philosophy of TPM, as TPM in itself is a technical process approach to achieve maintenance excellence. Workplace improvement was found to be positively related to extent of TPM implementation. This goes to show 
that Process-oriented strategy is also important in determining the extent of TPM implementation. Once the decision to adopt is made, there has to be an equal investment in the workplace improvement which the organization has to undertake in terms of equipment standardization and work instruction controls need to be carried out across the production floor. This finding is supported by the works of Suzuki (1994) and Shimbun (1995).

\subsection{Human-oriented Strategy and Process-oriented Strategy: Comparison}

The impact and effectiveness of human and process oriented strategies towards the extent of TPM is simultaneously measured in hypothesis 3 and Multiple Regression analysis showed that Human-oriented Strategy is having a greater impact compared to Process-oriented Strategy. For the reason that introduction and implementation of TPM are considered as one of the form of change management in the organisation where changes in work culture, process and management systems, organisational environment, and the individual perspective within the organisation are crucial to the enforcement of commitment, involvement and matured attitudes. When all these human related issues has been restored in the environment then only the technical skills and knowledge of maintenance have the foundation to maximize their effectiveness.

\section{CONCLUSION}

It can be concluded that the extent of both the human and process oriented strategies would lead to higher TPM implementation in the organisation. However, the impact of Human-oriented Strategy is found to be greater then Process-oriented Strategy in fostering higher extent of TPM implementation as the changes and adoption in the organisation are much more related to human issues. Thus the management has to balance both these strategies in order to achieve the maximal effect of implementation.

\section{REFERENCES}

Anita, S., (2000), The Extent of Total Productive Maintenance Implementation on A Company's Performance, (unpublished), MBA thesis, University Science of Malaysia, Penang.

Bamber, C. J., Sharp, J. M. \& Hides, M. T., (1999), "Factors Affecting Successful Implementation of Total Productive Maintenance. An UK Manufacturing Case Study Perspective", Journal of Quality in Maintenance Engineering, 5(3), 162-181.

Blanchard, B. S., (1997). An Enhanced Approach for Implementing Total Productive Maintenance In the Manufacturing Environment, Journal of Quality in Maintenance Engineering, 3(2), 69-80.

Chen, F., (1997) "Issue In the Continuous Improvement Process for Preventive Maintenance: Observations from Honda, Nippondenso and Toyota", Production and Inventory Management Journal, 38(4), 13-16.

Dunn, R. L., (1992), "Plant Engineering Management at John Deere", Plant Engineering, 46(1), 7882.

Fang, L. C., (2000), "Implementing TPM in Plant Maintenance: Some Organisational Barriers", International Journal of Quality \& Reliability Management, 17(9), 1003-1016.

Fredendall, L. D., Patterson, J. W., Kennedy, W. J. \& Griffin, T., (1997), "Maintenance: Modeling Its Strategic Impact", Journal of Managerial Issues, 9(4), 440-453. 
Nakajima, S., (1988), Introduction to TPM. Cambridge: Productivity Press.

Nakajima, S., (1989), TPM Development Program: Implementing Total Productive Maintenance, Cambridge MA: Productivity Press.

Norman, R. M., (1997), "Uptime: The True Measurement of Maintenance Productivity", Modern Casting; 87(5), 51-55.

Parzinger, M. \& Nath, R., (2000), "A Study of Relationship between Total Quality Management Implementation Factors and Software Quality", Total Quality Management, 11(3), 353-371.

Patterson, J. W., Kennedy, W. J. \& Fredendall, L. D., (1995), "Total Productive Maintenance Is Not For This Company", Production And Inventory Management Journal, 36(2), 61-64.

Scontrino, M. P., (1995), "TPM in Process Industry", Personnel Psychology, 48(2), 456-458.

Shimbun, N. K., (1995). TPM Case Studies, Portland OR: Productivity Press.

Suzuki, T., (1994), TPM in Process Industry, Portland OR: Productivity Press.

Thiagarajan, T. \& Zairi, M., (1997), "A Review Of Total Quality Management In Practice: Understanding The Fundamentals Through Examples Of Best Practice Applications - Part 1", The TQM Magazine, 9(4), 270-286.

Tsang, A. H. C. \& Chan, P. K., (2000), "TPM Implementation in China: A Case Study", International Journal of Quality \& Reliability Management, 17(2), 144-157.

Weeks, B., (1995), "Are We Ready for TQM? A Case Study", Production and Inventory Management Journal, 36(4), 27-32.

Yamashina, H., (2000), "Challenge to World Class Manufacturing", International Journal of Quality \& Realibility Management, 17(2), 132-143. 